\title{
Review on solid dispersion for suitable drug
}

Sachin Nivrutti Gadekar ${ }^{1 *}$, Jeevan R. Rajguru ${ }^{2}$, Dr. Subhash V. Deshmane ${ }^{1}$, Dr. Kailash R. Biyani $^{3}$

${ }^{1}$ Department of Pharmaceutics and ${ }^{3}$ Department of Pharmacology, Anuradha College of Pharmacy, Chikhli dist -Buldhana 443201

${ }^{2}$ Department of Pharmaceutics, AAEMF's Delight Institute of Pharmacy, Koregaon Bhima, Pune, M.S. 412216

Received: 08-08-2021 / Revised Accepted: 24-08-2021 / Published: 01-09-2021

\begin{abstract}
Solubility is the phenomenon of dissolution of solute in solvent to give a homogeneous system is one of the important parameters to achieve desired concentration of drug in systemic circulation for desired pharmacological response. There are many solubility techniques solid dispersion is one of the most important method for enhancing solubility of water insoluble drug. Solid dispersion is an effective way of improving the dissolution rate of poorly water-soluble drugs and hence its bioavailability. The water-soluble carriers used in preparation of solid dispersion enhance the dissolution rate of the poorly water-soluble drug. The review article focuses on the materials and methods of preparation, advantages, disadvantages and characterization of the solid dispersion.
\end{abstract}

Key words: Solid Dispersion, Bioavailability, Aqueous Solubility, Carrier, Polymers

\section{INTRODUCTION}

The oral route of administration is the safest, most economical and the most convenient way of giving medicines. The dosage forms for oral route include tablets, capsules, powders, mixtures, emulsions and gels. It is also called per oral. Oral route is most convenient because can be self-administered, pain free, easy to take and cheap compared to most other parenteral routes. Through oral route absorption takes place along the whole length of the gastro intestinal tract. Drug absorption from the gastrointestinal (GI) tract can be limited by a variety of factors with the most significant contributors being poor aqueous solubility and/or poor membrane permeability of the drug molecule. When delivering an active agent orally, it must first dissolve in gastric and/or intestinal fluids before it can then permeate the membranes of the GI tract to reach systemic circulation. Therefore, a drug with poor aqueous solubility will typically exhibit dissolution rate limited absorption, and a drug with poor membrane permeability will typically exhibit permeation rate limited absorption. The solubility

Address for Correspondence: Sachin Nivrutti Gadekar, Department of Pharmaceutics, Anuradha College of Pharmacy, Chikhli dist -Buldhana 443201; E-mail: jeevanrajguru97@gmail.com

How to Cite this Article: Sachin Nivrutti Gadekar, Jeevan R. Rajguru, Dr. Subhash V. Deshmane, Dr. Kailash R. Biyani. Review on solid dispersion for suitable drug. World J Pharm Sci 2021; 9(9): 230-235.

This is an open access article distributed under the terms of the Creative Commons Attribution-NonCommercialShareAlike 4.0 International License, which allows adapt, share and build upon the work non-commercially, as long as the author is credited and the new creations are licensed under the identical terms. (cc) EY-NC-SA 
behaviour of the drugs remains one of the most challenging aspects in formulation development \& it is key determinant to its oral bioavailability \& it is the rate limiting step to absorption of drugs from G.I.T. This results in important products not reaching the market or not achieving their full potential. Solid dispersion has attracted considerable interest as an efficient means of improving the dissolution rate \& bioavailability of hydrophobic drugs.

Hence, two areas of pharmaceutical research that focus on improving the oral bioavailability of active agents include: (i) enhancing solubility and dissolution rate of poorly water-soluble drugs and (ii) enhancing permeability of poorly permeable drugs. Numerous solid dispersion systems have been demonstrated in the pharmaceutical literature to improve the dissolution properties of poorly water-soluble drugs.

Solid Dispersion: In 1961, Sekiguchi and Obi first proposed the utilization of solid dispersions to increase the dissolution and oral absorption of poorly water-soluble drugs. They proposed the formation of a eutectic mixture of a poorly watersoluble drug with a physiologically inert, easily soluble carrier. ${ }^{[5]}$ Traditional methods suffer from the use of mechanical forces and excess organic solvents. In this technique, a poorly soluble drug is dispersed in a highly soluble solid hydrophilic matrix, which enhances the dissolution of the drug. Solid dispersion techniques can yield eutectic (non molecular level mixing) or solid solution (molecular level mixing) products.

Definition: The term solid dispersion refers to a group of solid products consisting of at least two different components, generally a hydrophilic matrix and a hydrophobic drug. The matrix can be either crystalline or amorphous. The drug can be dispersed molecularly, in amorphous particles (clusters) or in crystalline particles.

Types of Solid Dispersions: On the basis of molecular arrangement, different types of solid dispersions can be distinguished as follows

Eutectic mixtures: Two compounds which are completely miscible in the liquid state leads to simple eutectic mixture formation but only to a very limited extent in the solid state. This is usually prepared by solidification of fused melt of two components that shows complete liquid miscibility but negligible solid-solid solution. Fig. I (1).
Solid solution: In a solid solution, components crystallize together in a homogeneous one-phase system. The particle size of the drug in the solid solution is reduced to its molecular size. Thus a solid solution can achieve a faster dissolution rate than the corresponding eutectic mixture.

According to their miscibility two types of solid solution are:

i. Continuous Solid Solution: In a continuous solid solution, the components are miscible in all proportions. Theoretically, this means that the bonding strength between the two components is stronger than the bonding strength between the molecules of each of the individual components. Solid solutions of this type have not been reported in the pharmaceutical literature to date. Fig. I (2)

ii. Discontinuous Solid Solutions: In the case of discontinuous solid solutions, the solubility of each of the components in the other component is limited. A typical phase diagram, show the regions of true solid solutions. In these regions, one of the solid components is completely dissolved in the other solid component. Below a certain temperature, the mutual solubilities of the two components start to decrease. ${ }^{[11]}$ Fig. I (3)

According to the criteria of molecular size of the two components, the solid solutions are classified as:

i. Substitutional Crystalline Solutions: A substitutional crystalline solid dispersion is a type of solid solutions which have a crystalline structure, in which the solute molecules substitute for solvent molecules in the crystal lattice. Substitution is only possible when the size of the solute molecules differs by less than $15 \%$ or so from that of the solvent molecules. Fig. I (4) ii. Interstitial Crystalline Solid Solutions: In interstitial solid solutions, the dissolved molecules occupy the interstitial spaces between the solvent molecules in the crystal lattice. As in the case of substitutional crystalline solid solutions, the relative molecular size is a crucial criterion for classifying the solid solution type. In the case of interstitial crystalline solid solutions, the solute molecules should have a molecular diameter that is no greater than 0.59 of the solvent molecule's molecular diameter. Furthermore, the volume of the solute molecules should be less than $20 \%$ of the solvent. ${ }^{[11]}$ Fig. I (5). 

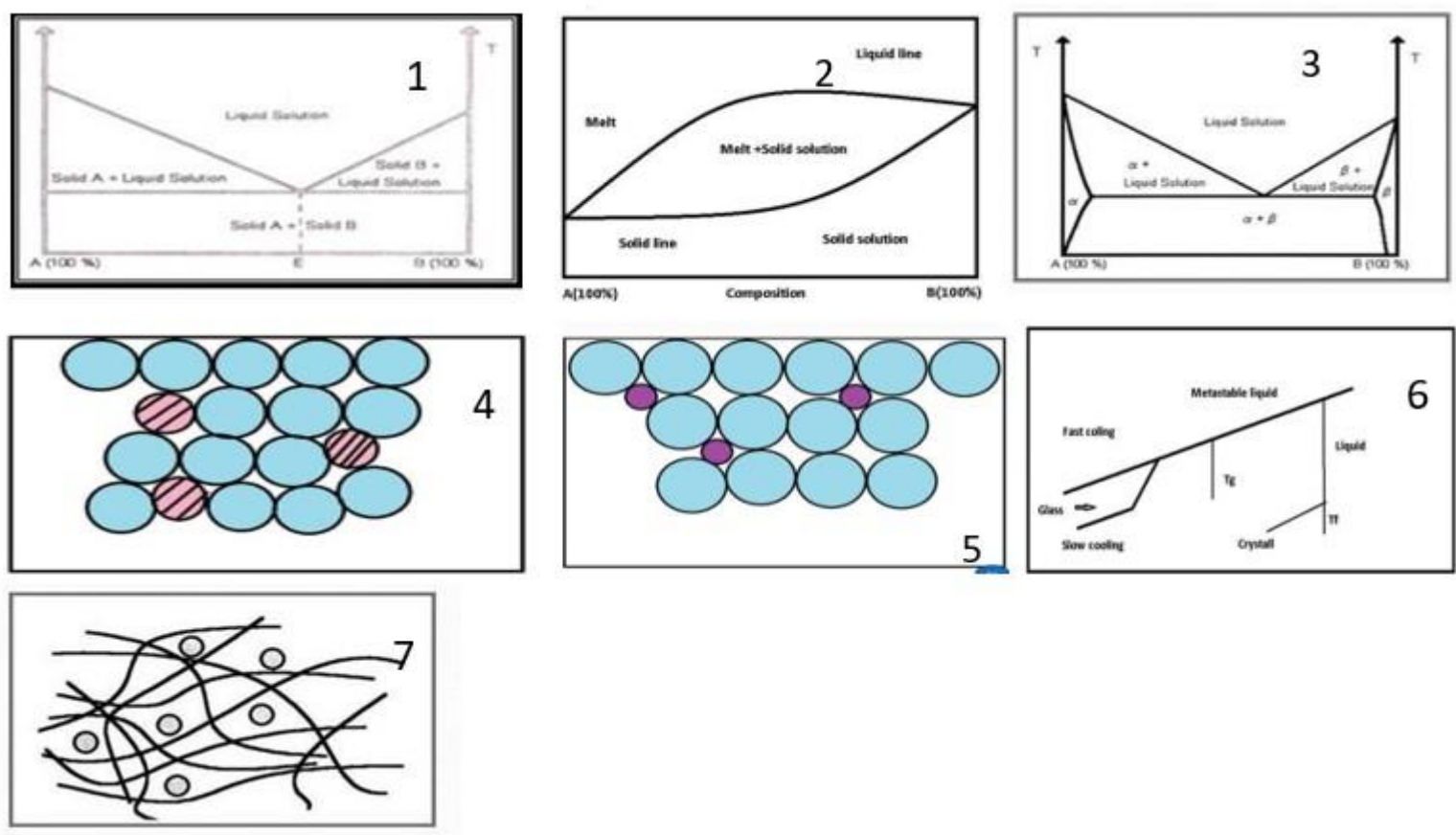

Figure I:I(1)-Eutectic Mixture, I(2)-Continuous Solid Solution, I(3)-Discontinuous Solid Solution, I(4)Substitutional Crystalline Solution, I(5)- Interstitial Crystalline Solution, I(6)-Glass Solution And Suspension, I(7)-Amorphous Precipitation in crystalline carrier.

Glass solutions and glass suspensions: Chiou and Riegelman first introduced the concept of formation of a glass solution as another potential modification of dosage forms in increasing drug dissolution and absorption. A glass solution is a homogenous, glassy system in which a solute dissolves in a glassy solvent. The familiar term glass however, can be used to describe either a pure chemical or a mixture of chemicals in a glassy or vitreous state. The glassy or vitreous state is usually obtained by an abrupt quenching of the melt. It is characterized by transparency and brittleness below the glass transition temperature eg. On heating, it softens progressively and continuously without a sharp melting point. ${ }^{[12]}$ Fig. I (6)

Amorphous precipitations in crystalline carrier: In the group of dispersion drug is precipitated out in amorphous form while in simple eutectic mixture it is in crystalline form. Fig. I (7)

Different methods of preparation of solid dispersion: There are various methods for preparation of solid dispersions. These methods deal with the challenge of mixing a matrix and a drug, preferably on a molecular level, while matrix and drug are poorly miscible. During many of the preparation techniques, de-mixing (partially or complete), and formation of different phases is observed. a. Fusion method: The melting or fusion method is the preparation of physical mixture of a drug and a water-soluble carrier and heating it directly until it melts. The melted mixture is then solidified rapidly in an ice-bath under vigorous stirring. The final solid mass is crushed, pulverized and sieved.

b. Solvent Evaporation method: Drug and carrier both are dissolved in organic solvent. The solvent is evaporated after dissolving the drug and carrier. The solid mass is ground, sieved and dried. A new method of solvent evaporation is Modified solvent evaporation method. In this method, the drug is dissolved with continuous stirring in an organic solvent at its saturation solubility. The polymer is suspended in a sufficient amount of water (up to the wet mass of the polymer). The drug solution is poured into the polymer suspension and the entire solvent is evaporated and the mass is obtained is dried.

c. Lyophilization technique: Lyophillization has been thought of a molecular mixing technique where the drug and carrier are co-dissolved in a common solvent, frozen and sublimed to obtain a lyophilized molecular dispersion.

d. Melt agglomeration on process: This technique has been used to prepare solid dispersions wherein the binder acts as a carrier. In addition, solid dispersions are prepared either by heating binder, drug and excipients to a temperature above the melting point of the binder (melt- in procedure) or 
by spraying a dispersion of drug in molten binder on the heated excipients (spray- on procedure) by using a high shear mixer.

e. Melt extrusion method: In this method, the drug and carrier are simultaneously mixed, heated, melted, homogenized and extruded in a form of tablets, rods, pellets, or milled and blended with other excipients for different purposes. The intense mixing and agitation forced by the rotating screw during the process cause disaggregation of drug particles in the molten polymer, resulting in a homogenous dispersion. This process involves the transformation of a solid mass of intertwined particles into a viscous liquid or semisolid mass by heating and intense mixing. The hot melt extruded systems are composed of drugs, one or more meltable polymers and other additives such as plasticizers and $\mathrm{pH}$ modifiers.

f. Supercritical Fluid Methods: Supercritical fluid methods are mostly applied with carbon dioxide (CO2), which is used as either a solvent for drug and matrix or as an anti-solvent. When supercritical $\mathrm{CO} 2$ is used as solvent, matrix and drug are dissolved and sprayed through a nozzle, into an expansion vessel with lower pressure and particles are immediately formed. The adiabatic expansion of the mixture results in rapid cooling. This technique does not require the use of organic solvents and since $\mathrm{CO} 2$ is considered environmentally friendly, this technique is referred to as 'solvent free'. The technique is known as Rapid Expansion of Supercritical Solution (RESS).

g. Electrostatic spinning: In this method, a drugpolymer solution is placed into a spinneret connected with a micro syringe pump and a high voltage between 5 and $30 \mathrm{kV}$ is applied to the needle tip to induce a charge on the surface of the solution. A fixed electrical potential is also applied across a fixed distance between the spinneret and the collector. When electrical forces overcome the surface tension of the feeding solution at the air interface, polymer jets are ejected. After coming out, the charged jets go straight for some distance, and then travel a spiral path because of the whipping instability. As the jet accelerates through the electric field, the solvent evaporates rapidly to make fibres at micron or submicron diameter which are collected on the screen or a spinning mandril. The collected fibres produce a non-woven fabric, which can be used in oral dosage forms by direct incorporation of the materials into a capsule or by further processing such as milling or grinding.

h. The use of surfactant: The utility of the surfactant systems in solubilization is well known. Adsorption of surfactant on solid surface can modify their hydrophobisity, surface charge, and other key properties that govern interfacial processes such as flocculation/dispersion, floatation, wetting, solubilization, detergency and enhanced oil recovery and corrosion inhibition. Surfactants have also been reported to cause solvation/plasticization, manifesting in reduction of melting the active pharmaceutical ingredients, glass transition temperature and the combined glass transition temperature of solid dispersions. Because of these unique properties, surfactants have attracted the attention of investigators for preparation of solid dispersions.

i. Dropping method: Solid dispersion of a melted drug-carrier mixture is pipette and then dropped onto a cooling plate, where it solidifies into round particles. The size and shape of the particles can be influenced by factors such as the viscosity of the melt and the size of the pipette. The dropping method does not use organic solvents and, therefore, has none of the problems associated with solvent evaporation.

j. Direct capsule filling: Direct filling of hard gelatin capsules with the liquid melt of solid dispersions avoids grinding-induced changes in the crystallinity of the drug. This molten dispersion forms a solid plug inside the capsule on cooling to room temperature, reducing cross contamination and operator exposure in a dust-free environment, better fill weight and content uniformity was obtained than with the powder-fill technique.

k. Gel entrapment technique: Carrier is dissolved in organic solvent to form a clear and transparent gel. Then drug is dissolved in gel by sonication for few minutes. Organic solvent is evaporated under vacuum. Solid dispersions are reduced in size by glass mortar and sieved.

l. Kneading method: Drug and each of surfaceactive carriers were weighed accurately in various ratios $(1: 1,1: 2)$ and transferred to china dish sufficient quantity of ethanol was added and the thick slurry was needed for $1 \mathrm{hr}$ and then dried at 450c until dryness. The dried mass was pulverized and sieved through sieve num \#120.the resulting solid dispersions were stored for $24 \mathrm{hrs}$ in desiccators to congeal. The mass obtained was crushed, pulverized. Finally dispersions were stored in air tight containers till further use.

\section{Advantages of solid dispersion:}

1. Reduce the particle size.

2. Improve the wettability and porosity.

3. Decrease the crystalline structure of drug in to amorphous form.

4. Improve dissolvability in water of a poorly water-soluble drug in a pharmaceutical. 
5. It helps to mask the taste of the drug substance.

6. It helps to formulate a faster release priming dose in a sustained release dosage form.

\section{Disadvantages of Solid Dispersion}

1. Major disadvantage is their instability.

2. They show changes in crystallinity and a decrease in dissolution rate with ageing.

3. Temperature and moisture have more deteriorating effect on solid dispersions than on physical mixtures.

4. Difficulty in handling because of tackiness

\section{Carriers}

\section{Selection of carriers:}

A carrier should meet the following criteria to be suitable for increasing the dissolution rate of a drug.

i. Freely water-soluble with intrinsic rapid dissolution properties.

ii. Non-toxic and pharmacologically inert.

iii. Heat stable with a low melting point for the melt method.

iv. Soluble in a variety of solvents and pass through a vitreous state upon solvent evaporation for the solvent method.

v. Able to preferably increase the aqueous solubility of the drug.

vi. Chemically compatible with the drug and not form a strongly bonded complex with the drug.

\section{Drug Carriers:}

i. Polymers: Polyvinylpyrrolidone, Polyvinylpolypyrrolidone, Polyvinylalcohol, Polyethylene glycols, Hydroxypropylmethylcellulose, Hydroxypropylcellulose, Poly (2hydroxyethyl methacrylate), Methacrylic copolymers.

ii. Cyclodextrins: $\quad \beta$-Cyclodextrins, Hydroxypropyl- $\beta$-cyclodextrins.

iii. Carbohydrates: Lactose, Soluble starch, Sorbitol, Mannitol.

iv. Surfactants: Poloxamers (Lutrol ${ }^{\circledR}$ F 127, Lutrol ${ }^{\circledR}$ F 68), Polyglycolized glyceride (Labrasol),Polyoxyethylene sorbitan monoesters (Tweens), Sorbitan esters (Spans), Polyoxyethylene stearates, Poly (beta-benzylL-aspartate) -b- poly (ethylene oxide), Poly (caprolactone) -b- poly(ethylene oxide).

v. Hydrotropes: Urea, Nicotinamide, Sodium benzoate, Sodium salicylate, Sodium acetate,
Sodium-o-hydroxybenzoate, Sodium phydroxy benzoate, Sodium citrate.

vi. Polyglycolized glycerides: Gelucire 44/14, Gelucire 50/13, Gelucire 62/05.

vii. Acids: Citric acid, Succinic acid, Phosphoric acid

viii. Miscellaneous: Microcrystalline cellulose, Dicalcium phosphate, Silica gel, Sodium chloride, Skimmed milk microcrystalline cellulose, Dicalcium phosphate, Silica gel, sodium chloride.

\section{Characterization of Solid Dispersions:}

i. Differential Scanning Calorimetry (DSC).

ii. Powder X-ray diffraction (PXRD).

iii. Fourier Transformed Infrared spectroscopy (FTIR).

iv. Dissolution studies.

v. Solubility Studies.

vi. Scanning Electron Microscopy (SEM)

vii. Drug content estimation.

\section{MATERIALS AND METHODS:}

Followings are the different materials are used in the preparation of solid dispersion:

I. Active Pharmaceutical Ingredient

II. Carriers: e.g. Polyvinylpyrrolidone, poloxamers, polyglycolized glycerides, hydrotopes.

III. Solvent: Methonol, ethanol, chloroform.

IV. Others: 1.2 Acetate Buffer, 6.8 Phosphate buffer, 7.4 Phosphate buffer.

The Various formulations of solid dispersions will prepared by any one or two following methods.

1. Fusion method.

2. Solvent evaporation method.

3. Kneading method.

\section{CONCLUSION}

Solid dispersion preliminary solubility analysis was carried out by using different carriers. The solid dispersion was prepared with D-mannitol, HPMC and PVP-K30. These solid dispersions were analyzed for the solubility and In vitro dissolution profile, solid dispersion of drug with had shown enhanced solubility with improved dissolution rate. HPMC, PVP K30, D-Mannitol improved the rate of dissolution indicating dissolution- efficiency and oral absorption in comparison to that observed with marketed-products of Ramipril. In present study solid dispersion prepared with selected carriers shows the presence of amorphous form confirmed by the characterization study.

\section{REFERENCE}

1. Al Nima Amina M, Al-Kotaji Myasar M, A preparation and evaluation of Meloxicam Solid Dispersion by Solvent Evaporation Method, International Research Journal of Pharmacy. 2014:5(11):838-845.

2. Barar F.S.K, Essentials of Pharmacotherapeutics. S. Chand Publications.2013, 5-10. 
3. Brahmankar D M, Jaiswal S B. Biopharmaceutics and Pharmacokinetics-A Treatise,Vallabh Prakashan.2015:5.

4. Chaulang G, Patil K, Preparation and Characterization of Solid Dispersion Tablet of Furesomide with Crospovidone. Research Journal Pharmaceutical and Technology.2008:1(4):386-389.

5. D Sonali, Thakkar S T, Silymarin-solid dispersions: Characterization and influence of preparation methods on dissolution .Acta Pharma Research Paper. 2010:(60): 427-443.

6. Deshmane Subhash, Shingne Nitin, Solubility Enhancement of Pioglitazone HCl Using Plain and Modified Xanthan Gum in Solid Dispersion, Novel Excipients 2015 Vol 2015, 378-386.

7. Handa U, Saroha K and Rana R. A review on: solid dispersions using carriers such as super-disinfectants and surfactants, World Journal of Pharmaceutical and life sciences.2016:2(3):171-188.

8. Ingle U. S, Gaikwad P.D. A Review on Solid Dispersion: A dissolution enhancement technique, International Journal of Research in Ayurveda and Pharmacy.2011:2(3):751-757.

9. Joshi M, Tiwari G, Review Article on Solid Dispersion: A Technology for the Improvement of Oral Bioavailability of Poorly Soluble Drugs, Current Pharma Research.2012:2(4): 668-677.

10. Kalia A, Poddar M, Review article on Solid Dispersions. An approach Towards Enhancing Dissolution Rate, International Journal of Pharmacy and Pharmaceutical Sciences.2011:3(4):9-19.

11. Katariya V R, Patil S B, Recent breakthroughs in Solid Dispersion. A Review, International Journal of Pharmaceutical Research \& Allied Sciences.2013:2(4):1-15.

12. Khatry S, Sood N Surface Solid Dispersion-A Review, International Journal of Pharmaceutical Science and Nanotechnology.2013:6(1):1915-1924.

13. Kurmi R, Mishra D K, Review article on Solid dispersion. A novel means of solubility enhancement.2016:3(1):1-8.

14. Luhadiya A, Agrawal S, A Review on Solid Dispersion, International Journal of Advanced Research in Pharmaceutical and Bio Sciences-2012; 2 (2): 281-291.

15. O G. Bhusnure, P A. Kazi, Research Article on Solid dispersion: an ever green method for solubility enhancement of poorly water soluble drug, International journal of research in pharmacy and chemistry. 2014, 4(4), 906-918.

16. Subhash Deshmane, Snehal Deshmane, Enhancement of solubility and bioavailability of ambrisentan by solid dispersion using Daucus carota as a drug carrier: formulation, characterization, in vitro, and in vivo study 11 Jan 2018 Vol 44 (06),1001-1011 